\title{
Thrombosis of the innominate artery during prolonged cardiac support by right transaxillary Impella $\mathbf{5 . 0}$ device
}

\author{
Aldo Cannata, Massimiliano Carrozzini, Giuseppe Tata, Claudio Francesco Russo \\ Department of Cardiac Surgery, Niguarda Hospital, Milan, Italy
}

\begin{abstract}
We describe an unexpected complication in a 46-years-old man affected by ischemic cardiomyopathy who received a prolonged mechanical cardiac support by means of right transaxillary Impella 5.0. The postoperative course was complicated by recurrent septicemia from multi-durg resistant Acinetobacter baumanii. He underwent implantation of venoarterial extracorporeal membrane oxygenation because of refractory cardiac arrest, followed by Impella removal that has been complicated by embolic stroke. The likely source of the embolus was a large thrombus inside the innominate artery. After a long-standing right transaxillary device implantation, particularly in patients with intercurrent septic episodes, a CT angiographic scan should be planned to exclude arterial thrombosis before removal of the transarterial device.
\end{abstract}

\section{Introduction}

Intravascular devices for mechanical cardiac support are gaining increasing diffusion because they are minimally invasive and reliable. Here we describe an unexpected complication following a

Correspondence: Aldo Cannata, Department of Cardiac Surgery, Niguarda Hospital, Piazza Ospedale Maggiore, 3, 20162 Milan, Italy. Tel.: +39.026444 2565 .

E-mail: aldo.cannata@ospedaleniguarda.it

Key words: Arterial thrombosis; thromboembolism; mechanical circulatory support; heart failure; sepsis.

Contributions: AC and MC wrote the text; GT reviewed the relevant literature; CFR reviewed the entire case report.

Conflict of interest: No one. This work was not supported by any grant.

Availability of data and materials: All data underlying the findings are fully available.

Ethics approval: No ethical commitee approval was required for this case report by the Department.

Received for publication: 20 May 2020:

Revision received: 21 October 2020.

Accepted for publication: 6 November 2020 .

This work is licensed under a Creative Commons Attribution 4.0 License (by-nc 4.0).

${ }^{\circ}$ Copyright: the Author(s), 2021

Licensee PAGEPress, Italy

Emergency Care Journal 2021; 17:9123

doi:10.4081/ecj.2021.9123 very long period of support by means of Impella 5.0 (Abiomed, Danvers, MA, USA) associated to recurrent sepsis.

\section{Case Report}

A 46-years-old Caucasian man was admitted to another Center due to a sub-acute myocardial infarction. Coronary angiogram showed severe multivessel disease. Stenting of the right coronary artery was performed. Nevertheless, left ventricular systolic function remained poor being ejection fraction $15 \%$. Because of refractory cardiogenic shock, a temporary mechanical cardiac support was instituted through a transfemoral Impella CP (Abiomed, Danvers, MA, USA). Impella is a minimally invasive pump that is positioned retrogradely into the left ventricle from a peripheral artery. It can be inserted percutaneously or surgically. It increases the left ventricular output by generating an axial flow of blood up to $5 \mathrm{~L} / \mathrm{min}$ from the left ventricle to the ascending aorta. The main indications to Impella are the support of a failing left ventricle, and during high-risk cardiac percutaneous interventions. Contraindications to it include significant aortic valve regurgitation or stenosis, a mechanical aortic valve prosthesis, a left ventricular thrombus and severe peripheral artery disease. After six days of support a malfunction of the device was detected due to inflow thrombosis. Therefore, Impella CP was removed and replaced with a right transaxillary Impella 5.0, surgically implanted through a vascular graft. The subsequent clinical course was complicated by septicemia due to Acinetobater baumanii. However, there was no signs of cardiac recovery and multiple attempts to wean the patient fromthe mechanical support resulted unsuccessful. During the circulatory support the level of anticoagulation remained within the therapeutic range. On the $52^{\text {nd }}$ day of Impella 5.0 support, the patient was referred to our hospital to evaluate the indication to heart transplantation. A relapse of septicemia by multi-drug-resistant $A$. baumanii occurred. Considering the long-standing Impella support and being very likely the infection of the device by a multidrug resistant pathogen, we decided to attempt a further weaning trial. However, it was complicated by a sudden refractory cardiac arrest, treated by means of reverting to Impella full support and an emergent institution of a femoral-femoral veno-arterial Extracorporeal Membrane Oxygenation (ECMO). Then the patient was brought in the operating room to remove the Impella. The procedure was preceded by transesophageal echocardiography, which excluded cardiac or device thrombosis. The Impella was surgically removed according to the standard procedure: the device was withdrawn and, after a brief blood flushing, the vessel was transiently clamped to allow for vascular graft removal and patch reconstruction of the axillary artery. Immediately after Impella extraction the near infrared spectroscopy brain monitoring showed a sudden drop in the right side signal. At the end of surgery the neurologic assessment of the patient revealed a left hemiplegia. Angio-CT scan showed distal occlusion of the right carotid artery, causing an extensive hemispheric stroke. 
A large molding thrombus was detected in the innominate artery (Figure 1). This massive stroke was the cause of the patient's death.

\section{Discussion}

Transaxillary implantation of Impella 5.0, either performed surgically of percutaneously, is gaining increasing acceptance as an effective method to unload the left ventricle..$^{1-5}$ A relevant advantage of this access is the possibility of a full mobilization of patients, being this particularly favourable in patients needing prolonged support awaiting heart transplantation. ${ }^{1}$ In some published series transaxillary implantation of Impella 5.0 was performed thorough the right axillary artery. ${ }^{2,3}$ Complications have been observed during support with Impella, such as vascular injury at the site of insertion (pseudoaneurysm, hematoma, thrombosis, limb ischemia), rupture of mitral chords, injury to the aortic valve leaflets, hemolysis and thrombocytopenia. To the best of our knowledge, this is the first report of a thrombosis of the innominate artery occurred during support with transaxillary Impella 5.0. The angio-CT scan showed a large molding thrombus inside the innominate artery. Its shape suggests that it was surrounding the Impella 5.0 catheter and it was the most likely source of the emboli to the right carotid artery (Figure 1). The reported incidence of stroke in patients supported by a transaxillary Impella 5.0 is $10 \% 0^{1,2}$ The risk of thrombosis of the innominate artery during support by means of right transaxillary Impella is still unknown. In our case the very long duration of the support with Impella and the hypercoagulable state associated to sepsis may have promoted the formation of intra-arterial thrombus. However, there are several published reports of patients successfully supported up to 35 days, ${ }^{3,6}$ far beyond the 14-days certification of Impella 5.0. Very recently Jones ${ }^{5}$ reported for the first time that the risk of thrombosis of the axillary artery was related to the duration of support by transaxillary Impella 5.0. The incidence of thrombosis was $21 \%, 67 \%$ and $75 \%$ for implant duration of 0-3, 4-7 and >7 days, respectively. Obviously, the clinical impact of thromboembolism from the innominate artery is very likely less benign as compared to a similar event arising from the axillary or the femoral artery because of the risk of stroke in the former case. The usual technique to remove a surgical transaxillary Impella is

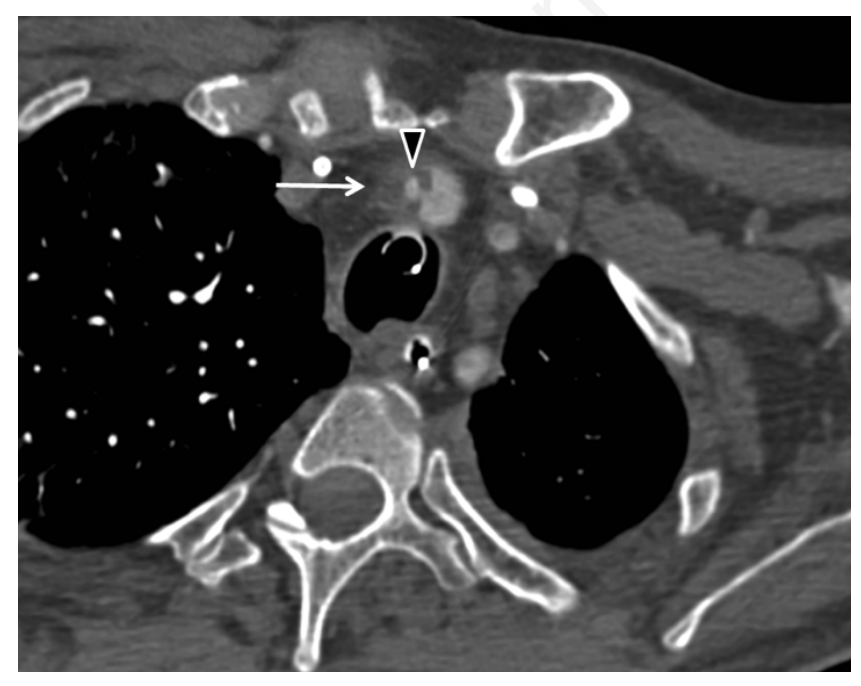

Figure 1. Angio-CT scan of the chest performed immediately after the removal of the Impella. It is shown a thrombus involving half the lumen of the innominate artery (white arrow). The catheter mold is visible into the thrombus (black arrowhead). by withdrawing the device without preoperative imaging. ${ }^{3}$ Pre-removal axillary artery angiography has been reported only for percutaneous transaxillary Impella. ${ }^{4,5}$ Recently Ranganath ${ }^{7}$ described a case of successful embolic protection of both carotid arteries during the removal of a transaxillary Impella by means of embolic protection devices. These were adopted prophylactically and the authors did not perform a pre-removal angio-CT scan. In our case antegrade advancement of an embolic protection device would have been very risky because of the thrombus in the innominate artery. Alternatively, a surgical thrombectomy of the innominate artery just before the Impella removal could have been considered. Nevertheless, it would have been at high risk of bleeding as a consequence of anticoagulation required by the venoarterial ECMO.

\section{Conclusions}

In conclusion, it seems advisable to perform an imaging study of the thoracic aorta and the involved epiaortic vessels just before the removal of a right transaxillary Impella 5.0, particularly for patients supported for more than 3 days, if the anticoagulation level has been suboptimal or in patients with recurrent sepsis. In case of thrombosis of the innominate artery the adoption of an embolic protection device or a surgical thrombectomy are potential options. Finally, it seems reasonable to prefer the implantation of the Impella through the left axillary artery whenever feasible, in order to decrease the risk of embolic carotid stroke.

\section{References}

1. Chung JS, Emerson D, Ramzy D, et al. A new paradigm in mechanical circulatory support: 100 patient experience. Ann Thorac Surg 2020;109:1370-7.

2. Boll G, Fischer A, Kapur NK, Salehi P. Right Axillary Artery Conduit Is a Safe and Reliable Access for Implantation of Impella 5.0 Microaxial Pump. Ann Vasc Surg 2019;54:54-9.

3. Bertoglio L, Katsarou M, Scandroglio M, et al. Surgical transaxillary placement of the Impella 5.0 ventricular assist device. J Card Surg 2019;34:92-8.

4. Nakamura K, Krishnan S, Mahr C, McCabe JM. First-in-Man Percutaneous Transaxillary Artery Placement and Removal of the Impella 5.0 Mechanical Circulatory Support Device. J Invasive Cardiol 2017;29:E53-9.

5. Jones TL, Kearney KE, McCabe JM. Prevalence and Predictors of Vascular Thrombus Formation After Percutaneous Axillary Artery Impella Insertion. Circ Cardiovasc Interv 2019;12:e008046.

6. Castillo-Sang MA, Prasad SM, Singh J, et al. Thirty-five day Impella 5.0 support via right axillary side graft cannulation for acute cardiogenic shock. Innovations (Phila) 2013;8:307-9.

7. Ranganath NK, Moazami N, Staniloae CS, Hisamoto K. Successful embolic protection during temporary circulatory support device removal in a patient who required holding of anticoagulation for postoperative hemothorax. J Thorac Cardiovasc Surg 2018;156:e203-4. 